\title{
Long strings and light scalar fields in the quark-gluon plasma
}

\author{
Henri Verschelde ${ }^{* \dagger}$, Valentin I. Zakharov \\ ${ }^{\dagger}$ Ghent University, Department of Physics and Astronomy, \\ Krijgslaan 281 - S9, 9000 Ghent, Belgium. \\ *Institute for Theoretical and Experimental Physics, \\ B. Cheremushkinskaya 25, Moscow, 117218, Russia. \\ Max-Planck-Institut für Physik, \\ Föhringer Ring 6, 80805 München, Germany.
}

E-mail: henri.verschelde@ugent.be, xxz@mppmu.mpg.de

\begin{abstract}
We review the physics of hot strings close to the Hagedorn temperature and their bearing on the deconfinement phase transition. We stress the importance of light scalar fields describing winding modes and their connection to the infrared degrees of freedom living on the stretched horizon. We comment on their relevance to holographic descriptions of the quark-gluon plasma and their relation with winding modes discovered on the lattice.
\end{abstract}

The many faces of $Q C D$

November 1-5, 2010

Gent Belgium

* Speaker. 


\section{Introduction}

Holographic description of gauge theories have provided new theoretical tools and new physical insights for the study of the quark-gluon plasma. In particular the strongly coupled but quasi ideal liquid nature of the plasma can be understood from the fluid/gravity duality [1] which tells us that strongly coupled liquids in gauge theory have a universal description as gravitational systems with $\eta / s=1 / 4 \pi$. On the other hand we would like to understand this behaviour of the strongly coupled quark-gluon plasma from a more fundamental gauge theory point of view. In this paper, we would like to point out the importance of light scalar fields. Our inspiration comes primarily from lattice results [2], which show that the deconfinement phase transition is driven by percolation of magnetic strings which change from $4 \mathrm{D}$ to $3 \mathrm{D}$. These percolation phase transitions can be described by the condensation of scalar fields living in the infrared and which vanish in the continuum limit. We show that these scalar fields have a natural holographic interpretation as fields describing winding modes around compact directions.

In section 2, we show how the statistical mechanics of hot strings close to the Hagedorn temperature can be captured by a thermal scalar field which is static and describes the winding mode around Euclidean time. In section 3, we briefly discuss the relevance of the Hagedorn phase transition to the holographic description of the deconfinement transition. In section 4, we derive (very much inspired by the work of Krusczenski [3]) the thermal scalar path integral, which is then used in section 5 to show that physical properties of a hot string gas such as the energy momentum tensor and the string charge have simple expressions in terms of a complex scalar field. The corresponding $U(1)$ symmetry is identified as coming from the Kaluza-Klein winding modes around Euclidean compactified time. In section 6, we propose an effective description of the stretched membrane of the AdS-black hole describing the plasma in terms of the thermal scalar field. Using the fluid/gravity duality which maps the fluid behaviour of the plasma on the fluid behaviour of the stretched membrane, we argue for the importance of the thermal scalar in the interpretation of the behaviour of the quark-gluon plasma. We make this connection even more plausible by discussing the Hawking-Page transition in the Witten model [15], from the point of view of condensation of winding modes as described by scalar fields. We interpret this phase transition as a transition from a 4D quantum condensed scalar field to a 3D condensed thermal scalar field and interpret this in terms of percolation transitions as observed on the lattice [2]. We end with some conclusions.

\section{The Hagedorn phase transition and the thermal scalar}

The Hagedorn phase transition in string theory is due to the well known fact that the density of states for highly energetic strings grows exponentially as:

$$
\rho(E) \sim \frac{e^{\beta_{H} E}}{E^{1+\alpha}}
$$

where

$$
\beta_{H}=4 \pi \alpha^{\prime 1 / 2}
$$

is the inverse Hagedorn temperature and $\alpha$ is determined by the number of non-compact dimensions. The divergence of the partition function for $\beta<\beta_{H}$ signals a limiting temperature which 
has the following physical interpretation [4]: the energy of a long string is given by $E=\left(\frac{N}{\alpha}\right)^{1 / 2}$ for large $N$, where $N$ is the total number operator. The number of states as a function of $N$ goes roughly as $\exp \left(4 \pi N^{1 / 2}\right)$ so that the entropy for long strings is given by $S=4 \pi \alpha^{1 / 2} E$.

The divergence of the partition function at $\beta=\beta_{H}$ means that the microcanonical ensemble is dominated by a single long string with constant temperature $\beta(E)=\frac{\partial S}{\partial E}=4 \pi \alpha^{1 / 2}$. So any energy that is pumped in the system goes into making the string longer, but does not change the temperature.

To relate this divergence to the existence of a light scalar field, we first show how we can rewrite the free energy of a free gas of relativistic particles as a path integral of a single particle winding around Euclidean time. We start from

$$
\begin{aligned}
F & =\frac{V}{\beta} \int \frac{\mathrm{d}^{D-1} k}{(2 \pi)^{D-1}} \ln \left(1-\exp \left(-\beta \omega_{k}\right)\right) \\
& =-\frac{V}{\beta} \sum_{w=1}^{\infty} \int \frac{\mathrm{d}^{D-1} k}{(2 \pi)^{D-1}} \frac{1}{w} \exp \left(-w \beta \omega_{k}\right)
\end{aligned}
$$

and use the identity

$$
\frac{1}{w} \exp (-w \beta \omega)=\frac{\beta}{\sqrt{2 \pi}} \int_{0}^{\infty} \frac{\mathrm{d} s}{s^{3 / 2}} \exp \left(-\frac{1}{2}\left(\omega s-\frac{w^{2} \beta^{2}}{s}\right)\right)
$$

and $\omega_{k}=\sqrt{k^{2}+m^{2}}$ to turn the integration over momenta into a Gaussian and finally obtain

$$
F=-V \sum_{w=1}^{\infty} \int_{0}^{\infty} \frac{\mathrm{d} s}{s(2 \pi s)^{D / 2}} \exp \left(-\frac{m^{2} s}{2}-\frac{w^{2} \beta^{2}}{2 s}\right)
$$

This equation for the free energy can be written as a one-particle path integral on $\mathbb{R}^{3} \times S_{1}^{\beta}$ by interpreting $s$ as the Schwinger parameter and $w$ as the winding number of classical trajectories $X_{0}^{\mathrm{cl}}(\tau)=w \beta \frac{\tau}{s}$ winding around Euclidean time:

$$
\beta F=-\int\left[\mathscr{D} X^{\mu}\right] \exp \left(-\frac{1}{2} \int \mathrm{d} \tau\left[\left(\frac{\partial X^{\mu}}{\partial \tau}\right)^{2}+m^{2}\right]\right) .
$$

Using the previous trick for all mass levels of the closed string, Polchinski [5] showed that the free energy of a free string gas can be written as the path integral of a single string on a torus in the Euclidean background $M \times S_{1}^{\beta}$

$$
\beta F=-\int_{0}^{\infty} \frac{\mathrm{d} \tau_{2}}{2 \tau_{2}} \int_{-\frac{1}{2}}^{+\frac{1}{2}} \mathrm{~d} \tau_{1} \Delta_{\mathrm{FP}} \int\left[\mathscr{D} X^{\mu}\right] \sqrt{G} \exp \left(-\frac{1}{4 \pi \alpha^{\prime}} \int \mathrm{d}^{2} \sigma \sqrt{h} h^{\alpha \beta} \partial_{\alpha} X^{\mu} G_{\mu \nu} \partial_{\beta} X^{v}\right)
$$

where $h_{\alpha \beta}$ is the worldsheet metric for a torus with moduli $\tau_{1}$ and $\tau_{2}$, and $G_{\mu \nu}$ is the background metric. For the compact time dimension, we have the following periodicity property:

$$
\begin{aligned}
& X^{0}\left(\sigma_{1}+1, \sigma_{2}\right)=X^{0}\left(\sigma_{1}, \sigma_{2}\right) \\
& X^{0}\left(\sigma_{1}, \sigma_{2}+1\right)=X^{0}\left(\sigma_{1}, \sigma_{2}\right)+w \beta, \quad w \in \mathbb{Z} .
\end{aligned}
$$

Winding number $w=0$ corresponds to vacuum energy. Note that the path integral (2.7) with the periodicity conditions (2.8) is not explicitly invariant under modular transformations. It was shown 
in [6] that (2.7) can be made manifestly modular invariant by restricting to the fundamental modular domain $F_{0}$ with $-\frac{1}{2} \leq \operatorname{Re}[\tau] \leq \frac{1}{2},|\tau| \geq 1$ and imposing periodic boundary conditions

$$
\begin{array}{ll}
X^{0}\left(\sigma_{1}+1, \sigma_{2}\right)=X^{0}\left(\sigma_{1}, \sigma_{2}\right)+n \beta, & n \in \mathbb{Z} \\
X^{0}\left(\sigma_{1}, \sigma_{2}+1\right)=X^{0}\left(\sigma_{1}, \sigma_{2}\right)+w \beta, & w \in \mathbb{Z} .
\end{array}
$$

The Hagedorn temperature can now be understood as the temperature where a new light scalar appears. Indeed, the mass spectrum for closed strings winding around $S_{1}^{\beta}$ is given by:

$$
M^{2}=\frac{n^{2}}{R^{2}}+\frac{w^{2} R^{2}}{\alpha^{\prime 2}}+\frac{2}{\alpha^{\prime}}(h+\bar{h}-2)
$$

where $R=\beta / 2 \pi$. The scalar state $(h=\bar{h}=0)$ with Kaluza-Klein momentum $n=0$ and winding $w= \pm 1$ has mass:

$$
M^{2}(\beta)=\frac{\beta^{2}-\beta_{H}^{2}}{4 \pi^{2} \alpha^{\prime 2}}
$$

and becomes a massless complex $(w= \pm 1)$ scalar in $D-1$ dimensions at $\beta=\beta_{H}$ [7]. The dominance of the microcanonical ensemble by a single long string is here translated into an infrared divergence due to the so called thermal scalar. One can use the thermal scalar to derive the density of states at high energy. Indeed, for $\beta \sim \beta_{H}$, one has:

$$
\begin{aligned}
\beta F & \approx-V \int \frac{\mathrm{d}^{D-1} k}{(2 \pi)^{D-1}} \ln \left(k^{2}+M^{2}(\beta)\right) \\
& \approx V \int \frac{\mathrm{d}^{D-1} k}{(2 \pi)^{D-1}} \int_{0}^{\infty} \frac{\mathrm{d} E}{E} e^{-\beta E+\beta_{H} E-2 \pi^{2} \alpha^{2} k^{2} E / \beta_{H}} \\
& \approx \int_{0}^{\infty} \mathrm{d} E e^{-\beta E} \rho(E)
\end{aligned}
$$

with

$$
\rho(E)=V \frac{\beta_{H}^{(D-1) / 2}}{\left(4 \pi^{2} \alpha^{\prime}\right)^{D-1}} \frac{e^{\beta_{H} E}}{E^{1+(D-1) / 2}} .
$$

\section{The Hagedorn phase transition and deconfinement}

It was suggested in [8] that the Hagedorn temperature is not a limiting temperature but points to a first order phase transition with large latent heat similar to the deconfinement phase transition. Let us first discuss the deconfinement phase transition for pure Yang-Mills theory. For $T<T_{c}$, glueballs are thermally excited out of the vacuum and contribute $O(1)$ to the free energy. Above $T_{c}$, we have a gluon plasma which contributes $O\left(N c^{2}\right)$ to the free energy. From the standpoint of holography, the phase transition is a geometric Hawking-Page transition from a thermal AdS-space (AdS-metric with compactified time) to an AdS-space with a black brane. The classical thermal AdS-metric only contributes to the vacuum energy. So we have to go to one-loop or genus one (torus) partition function to get a contribution to the free energy which is therefore $O(1)$. Above $T_{c}$, the black brane metric contributes at the classical level (genus zero) to the free energy which now becomes $O\left(N c^{2}\right)$. This change from genus one to genus zero points to a quantum field which gets a classical $O\left(1 / g_{s}^{2}\right)$ expectation value. A similar scenario happens in the Hagedorn phase 
transition [8] where according to (2.11) the thermal scalar becomes tachyonic above $T_{H}$ and gets an expectation value. Similar phase transitions related to topology change in spacetime have been associated to condensation of thermal winding modes [9].

\section{Thermal scalar path integral}

The Hagedorn divergence is due to the $\tau_{2} \rightarrow 0$ (ultraviolet) behaviour of the torus path integral (2.7), where $\tau_{2}$ plays the role of Schwinger parameter. Indeed, in flat space we have:

$$
\beta F=\beta V_{D-1} \sum_{w= \pm 1}^{\infty} \int_{0}^{\infty} \frac{\mathrm{d} \tau_{2}}{2 \tau_{2}} \int_{-\frac{1}{2}}^{+\frac{1}{2}} \mathrm{~d} \tau_{1}|\eta(\tau)|^{4-2 d}\left(4 \pi \alpha^{\prime} \tau_{2}\right)^{-d / 2} \exp \left(-\frac{w^{2} \beta^{2}}{4 \pi \alpha^{\prime} \tau_{2}}\right)
$$

where $w$ is the thermal winding number and with

$$
\eta(\tau)=q^{1 / 24} \prod_{n=1}^{\infty}\left(1-q^{n}\right)
$$

and

$$
q=e^{i 2 \pi \tau}
$$

Since the integrand is largest when $\tau_{1}=0$ and

$$
|\eta(\tau)|^{2} \tau_{2} \stackrel{\left(\tau_{1}=0, \tau_{2} \rightarrow 0\right)}{\sim} \frac{1}{\tau_{2}} \exp \left(-\frac{\pi}{6 \tau_{2}}\right)
$$

the integrand in the torus partition function behaves as:

$$
\tau_{2}^{d / 2-3} \exp \left(-\frac{\pi(2-d)}{6 \tau_{2}}\right) \exp \left(-\frac{w^{2} \beta^{2}}{4 \pi \alpha^{\prime} \tau_{2}}\right)
$$

For $D=26$ and $w= \pm 1$, this is converging for $\tau_{2} \rightarrow 0$ if

$$
\frac{\beta^{2}}{4 \pi \alpha^{\prime}} \geq 4 \pi \quad \text { or } \quad \beta \geq \beta_{H}=4 \pi \alpha^{1 / 2} .
$$

The essential idea of the thermal scalar is that the ultraviolet divergence for $\tau_{2} \rightarrow 0$ can be described through some UV/IR connection as an infrared divergence for $\tau_{2} \rightarrow \infty$. For this, we use modular invariance of the torus partition function on a compact space [10]:

$$
\int[\mathscr{D} X] e^{-S(X)}=2 \pi R\left(4 \pi \alpha^{\prime} \tau_{2}\right)^{-1 / 2}|\eta(\tau)|^{-2} \sum_{n, w} \exp \left(-\frac{\pi R^{2}|n \tau-w|^{2}}{\tau_{2} \alpha^{\prime}}\right)
$$

under

$$
\left\{\begin{array}{l}
\tau \rightarrow \tau^{\prime}=1 / \tau \\
(n, w) \rightarrow\left(n^{\prime}, w^{\prime}\right)=(-w, n)
\end{array}\right.
$$

For $\tau_{1}=0$ and the periodicity conditions (2.8) this becomes

$$
\left\{\begin{array}{l}
\tau_{2} \rightarrow 1 / \tau_{2} \\
(0, w) \rightarrow(-w, 0)
\end{array}\right.
$$


So qualitativily, the ultraviolet divergence becomes an infrared divergence and the string wraps the thermal circle along the spatial worldsheet coordinate $\sigma_{1}$ :

$$
\begin{aligned}
& X^{0}\left(\sigma_{1}, \sigma_{2}+1\right)=X^{0}\left(\sigma_{1}, \sigma_{2}\right) \\
& X^{0}\left(\sigma_{1}+1, \sigma_{2}\right)=X^{0}\left(\sigma_{1}, \sigma_{2}\right)+w \beta
\end{aligned}
$$

Introducing the worldsheet reparametrization:

$$
\begin{gathered}
\sigma=\frac{\sigma_{1}}{\tau_{2}}, \quad \tau=\sigma_{2} \\
X\left(\sigma_{1}, \sigma_{2}\right) \rightarrow X(\sigma, \tau)
\end{gathered}
$$

we can obtain the dominant contribution in the infrared $\left(\tau_{2} \rightarrow \infty\right)$ by viewing $\tau_{2}$ as a "worldsheet temperature" and doing a high temperature expansion in Matsubara modes:

$$
\begin{aligned}
& X^{i}(\sigma, \tau)=\sum_{n=0}^{\infty} e^{i\left(2 \pi n \tau_{2}\right) \sigma} X_{n}^{i}(\tau) \\
& X^{0}(\sigma, \tau)= \pm \beta \tau_{2} \sigma+\sum_{n=0}^{\infty} e^{i\left(2 \pi n \tau_{2}\right) \sigma} X_{n}^{0}(\tau)
\end{aligned}
$$

where we have explicitly implemented $w= \pm 1$. In the $\tau_{2} \rightarrow \infty$ limit only the $n=0$ mode survives, and we get "dimensional reduction" from a string theory to a particle theory. The discarded oscillator modes however contribute to the worldsheet vacuum energy and this vacuum energy, which is

$$
\Delta S=-4 \pi \tau_{2}=-\frac{1}{4 \pi \alpha^{\prime} \tau_{2}}\left(\tau_{2}^{2} \beta_{H}^{2}\right),
$$

should be reintroduced by hand (just as the ultraviolet divergences coming from the nonzero Matsubara modes have to be explicitly added to recover the Stefan-Boltzmann contribution to the free energy for thermal quantum field theory). Adding all the relevant contributions, we obtain the particle action for the long string with thermal winding number $w= \pm 1[3]$ :

$$
\begin{aligned}
S_{\text {part }}=\frac{1}{4 \pi \alpha^{\prime}} & {\left[\beta^{2} \frac{\left(\tau_{1}^{2}+\tau_{2}^{2}\right)}{\tau_{2}^{2}} \int_{0}^{\tau_{2}} \mathrm{~d} \tau G_{00}-\beta_{H}^{2} \tau_{2}\right.} \\
& \left.\mp 2 \frac{\tau_{1}}{\tau_{2}} \beta \int_{0}^{\tau_{2}} \mathrm{~d} t G_{0 \mu} \partial_{t} X^{\mu}+\int_{0}^{\tau_{2}} \mathrm{~d} t G_{\mu \nu} \partial_{t} X^{\mu} \partial_{t} X^{v}\right]
\end{aligned}
$$

where the particle coordinates $X^{\mu}(\tau)$ are defined as Matsubara zero modes:

$$
X_{0}^{i}(\tau)=X^{i}(\tau), \quad X_{0}^{0}(\tau)=X^{0}(\tau)
$$

and we introduced $t=\tau_{2} \tau$.

For constant $G_{00}, G_{0 i}$ the linear term drops out due to the periodicity properties and the free energy due to the thermal winding modes $w= \pm 1$ is given by ${ }^{1}$ :

$$
\begin{aligned}
& \beta F=-2 \int_{0}^{\infty} \frac{\mathrm{d} \tau_{2}}{2 \tau_{2}} \int_{-\infty}^{+\infty} \mathrm{d} \tau_{1} \exp \left(-\frac{\tau_{1}^{2} \beta^{2} G_{00}}{4 \pi \alpha^{\prime} \tau_{2}}\right) \\
& \int\left[\mathscr{D} X^{\mu}\right] \sqrt{G} \exp \left(-\frac{1}{4 \pi \alpha^{\prime}}\left[\tau_{2}\left(\beta^{2} G_{00}-\beta_{H}^{2}\right)+\int_{0}^{\tau_{2}} \mathrm{~d} t G_{\mu \nu} \partial_{t} X^{\mu} \partial_{t} X^{\nu}\right]\right)
\end{aligned}
$$

\footnotetext{
${ }^{1}$ The integration region for $\tau_{1}$ is more complicated than in (4.16) and only valid for large $\tau_{2}$.
} 
The factor of 2 comes from $w= \pm 1$. For $G_{0 i}=0, G_{00}$ is constant, we can exactly do the $X^{0}$ path integral which gives a factor $\left(\frac{G_{00} \beta^{2}}{4 \pi \alpha^{\prime} \tau_{2}}\right)^{1 / 2}$. The factor $\sqrt{G_{00}}$ from the measure is crucial for this; the integration over $\tau_{1}$ gives a factor which is exactly the inverse so they cancel. Defining the Schwinger parameter $s=2 \pi \alpha^{\prime} \tau_{2}$ we find:

$$
\begin{aligned}
\beta F & =-\int_{0}^{\infty} \frac{\mathrm{d} s}{s} \int[\mathscr{D} X]_{D-1} \sqrt{G_{D-1}} \exp \left(-\frac{1}{2} \int_{0}^{s} \mathrm{~d} t\left[M^{2}(\beta)+G_{i j} \partial_{t} X^{i} \partial_{t} X^{j}\right]\right) \\
& =-\operatorname{Tr} \ln \left[\Delta+M^{2}(\beta)\right]
\end{aligned}
$$

where

$$
M^{2}(\beta)=\frac{\beta^{2} G_{00}-\beta_{H}^{2}}{4 \pi^{2} \alpha^{\prime 2}}
$$

and $\Delta$ is the Laplace operator in a $D-1$ dimensional space with metric $G_{i j}$. The physical interpretation is that the statistics of long strings are those of random walks in $D-1$ space dimensions [3].

\section{Static correlators and the physical significance of the thermal scalar}

The thermal scalar provides a physical picture of long strings as random walks in $D-1$ space dimensions. To investigate whether the thermal scalar is merely a mathematical device that allows calculation of the free energy, or has a deeper physical significance, we calculate some static correlators of physical quantities and show that they have a simple interpretation in terms of the thermal scalar. Let's take as an exemple the correlator $<T_{i j}\left(\vec{x}_{1}\right) T_{k l}\left(\vec{x}_{2}\right)>$ with $i \neq j, k \neq l$ and $T_{i j}(\vec{x})$ is the time averaged stress tensor at position $\vec{x}$ in $D-1$ dimensional space given by:

$$
T_{i j}(\vec{x})=\frac{1}{2 \pi \alpha^{\prime}} \int \mathrm{d}^{2} \sigma \sqrt{h} h^{\alpha \beta} \partial_{\alpha} X^{i} \partial_{\beta} X^{j} \delta^{D-1}\left(\vec{X}\left(\sigma_{1}, \sigma_{2}\right)-\vec{x}\right) .
$$

Retaining only the Matsubara zero modes in (4.12) and using symmetric point splitting to define the composite operators we obtain on the torus:

$$
T_{i j}(\vec{x})=\lim _{\varepsilon \rightarrow 0} \frac{1}{4 \pi \alpha^{\prime}} \int_{0}^{\tau_{2}} \mathrm{~d} t\left(\partial_{t} X^{i}(t-\varepsilon) \partial_{t} X^{j}(t+\varepsilon)+(i \leftrightarrow j)\right) \delta^{D-1}(\vec{X}(t)-\vec{x})
$$

Plugging $T_{i j}\left(\vec{x}_{1}\right) T_{k l}\left(\vec{x}_{2}\right)$ in the path integral (4.17) for $G_{i j}=\delta_{i j}$, and using at fixed $\tau_{2}$ :

$$
\begin{aligned}
& \left\langle\delta\left(\vec{X}\left(t_{1}\right)-\vec{x}_{1}\right) \delta\left(\vec{X}\left(t_{2}\right)-\vec{x}_{2}\right)\right\rangle_{\tau_{2}} \\
& \quad=\theta\left(t_{1}-t_{2}\right)\left\langle\vec{x}_{2}\left|e^{-\left|t_{2}-t_{1}\right| \pi \alpha^{\prime}\left(\Delta+M^{2}(\beta)\right)}\right| \vec{x}_{1}\right\rangle\left\langle\vec{x}_{1}\left|e^{-\left(\tau_{2}-\left|t_{1}-t_{2}\right|\right) \pi \alpha^{\prime}\left(\Delta+M^{2}(\beta)\right)}\right| \vec{x}_{2}\right\rangle+\left(t_{1} \leftrightarrow t_{2}\right)
\end{aligned}
$$

and the correspondence $\partial_{t} X^{i} \rightarrow-i 2 \pi \alpha^{\prime} \nabla_{i}$, we find after integrating over $t_{1}, t_{2}$, and $\tau_{2}$ :

$$
\left\langle T_{i j}\left(\vec{x}_{1}\right) T_{k l}\left(\vec{x}_{2}\right)\right\rangle=2 \nabla_{i}^{1} \nabla_{k}^{2}\left\langle\vec{x}_{1}\left|\frac{1}{\Delta+M^{2}(\beta)}\right| \vec{x}_{2}\right\rangle \nabla_{j}^{1} \nabla_{l}^{2}\left\langle\vec{x}_{1}\left|\frac{1}{\Delta+M^{2}(\beta)}\right| \vec{x}_{2}\right\rangle+(k \leftrightarrow l)
$$

This coincides with the one-loop expectation value of the stress tensor of a $D-1$ dimensional scalar with mass $M(\beta)$ given by

$$
T_{i j}=\partial_{i} \phi \partial_{j} \phi^{*}+(i \leftrightarrow j)
$$


for $i \neq j$.

To calculate static correlators of momentum density $T_{0 i}$, one has to start from the partition function (4.16). A detailed calculation [11] shows that, to leading order, a stringy contribution coming from the winding solution $X_{0}(\sigma, \tau)= \pm \beta \tau_{2} \sigma$ cancels exactly the contributions from the fluctuating coordinates $X_{0}(\tau)$ and $X_{i}(\tau)$.

Finally we can look at static correlators for the string charge which couples to the KalbRamond field. The corresponding conserved antisymmetric tensor current is given by:

$$
J^{\mu v}(x)=\frac{1}{2 \pi \alpha^{\prime}} \int \mathrm{d}^{2} \sigma \partial_{a} X^{\mu} \partial_{b} X^{v} \varepsilon_{a b} \delta^{D}\left(X^{\rho}-x^{\rho}\right) .
$$

Close to the Hagedorn temperature we can restrict ourselves to the lowest Matsubara mode in (4.12) so that $J_{i j}=0$ and the string charge averaged over time is given by:

$$
J_{0 i}(\vec{x})= \pm \frac{\beta}{2 \pi \alpha^{\prime}} \int_{0}^{\tau_{2}} \mathrm{~d} t \frac{\partial X^{i}}{\partial t} \delta^{(D-1)}(\vec{X}(t)-\vec{x}) .
$$

Plugging this into (4.16) and using point splitting, we find

$$
\begin{aligned}
\left\langle J_{0 i}\left(\vec{x}_{1}\right) J_{0 j}\left(\vec{x}_{2}\right)\right\rangle=-\frac{1}{2}\left(\frac{\beta}{\pi \alpha^{\prime}}\right)^{2} & {\left[\nabla_{i}^{1}\left\langle\vec{x}_{1}\left|\frac{1}{\Delta+M^{2}(\beta)}\right| \vec{x}_{2}\right\rangle \nabla_{j}^{2}\left\langle\vec{x}_{1}\left|\frac{1}{\Delta+M^{2}(\beta)}\right| \vec{x}_{2}\right\rangle\right.} \\
& \left.-\left\langle\vec{x}_{1}\left|\frac{1}{\Delta+M^{2}(\beta)}\right| \vec{x}_{2}\right\rangle \nabla_{i}^{1} \nabla_{j}^{2}\left\langle\vec{x}_{1}\left|\frac{1}{\Delta+M^{2}(\beta)}\right| \vec{x}_{2}\right\rangle\right]
\end{aligned}
$$

which gives the string charge in terms of the thermal scalar as

$$
J_{0 i}=\frac{i}{2}\left(\frac{\beta}{\pi \alpha^{\prime}}\right)\left[\phi \nabla_{i} \phi^{*}-\phi^{*} \nabla_{i} \phi\right]
$$

Note that this charge, which is tangent to the string, is conserved as a $D-1$ dimensional $U(1)$ current in the same way as electric current along a wire is conserved in magnetostatics. This $U(1)$ is a stringy effect due to winding around compactified time. Indeed, compactification leads to a $U(1) \times U(1)$ symmetry where the first $U(1)$ corresponds with Kaluza-Klein momentum and the second to winding number.

\section{The thermal scalar and the stretched membrane}

The stretched membrane has been introduced (for relevant literature, see [12]) as a physically intuitive picture to describe the response of a black hole to external perturbations. The black hole reacts to electromagnetic and gravitational perturbations as a charged and fluid membrane with physical properties such as entropy, conductivity and viscosity. The membrane hovers above the horizon at a distance $\sim l_{s}$ and for FIDO's (fiducial observers at fixed distance from the horizon) the membrane is at a temperature of the order of $T_{H}$. In a sense, the stretched membrane describes the collapsing process towards the black hole at asymptotically late times. The exact physical nature of this membrane is however still a mystery.

One could imagine a spherical shell of closed strings collapsing under its weight in a thermal AdS space and forming an AdS-black hole. At very late times, this shell would hover above the 
horizon and be at temperature $T_{\text {S.M. }} \sim\left(g_{00}\right)^{-1 / 2} T$. As the temperature would be of the order of $T_{H}$, the membrane could be effectively described as a long string. This picture of the stretched membrane was originally proposed by Susskind in [13] and points to the conclusion that the stretched membrane is described by the thermal scalar. Using holography, this means that the hydrodynamical properties of strongly interacting gauge theories could be understood in terms of light scalar fields describing long strings.

One particular aspect of the statistics of long strings close to the horizon is nicely illustrated by the thermal scalar picture. The string charge $J_{0 n}$ gives the density of strings piercing through a plane with normal vector $\vec{n}$. Close to the horizon, the local temperature approaches $T_{H}$ and the thermal scalar becomes massless. At the same time the correlator $\left\langle J_{0 n}\left(\vec{x}_{1}\right) J_{0 n}\left(\vec{x}_{2}\right)\right\rangle$ becomes long range which points to a percolation (through the plane) phase transition. This connection between condensates of winding modes and percolation will be used in the next section where we discuss the relevance of light scalar fields for the quark-gluon plasma.

\section{Long strings, thermal scalar and the quark-gluon plasma}

To relate long strings to the quark-gluon plasma, let us concentrate on the holographic model introduced by Witten [14] and which at finite temperature is in the same universality class as pure Yang-Mills theory. For $T<T_{c}$, we have the metric:

$$
\mathrm{d} s^{2}=\left(\frac{u}{R}\right)^{3 / 2}\left[\mathrm{~d} t^{2}+\delta_{i j} \mathrm{~d} x^{i} \mathrm{~d} x^{j}+f(u) \mathrm{d} x_{4}^{2}+\left(\frac{R}{u}\right)^{3 / 2}\left(\frac{\mathrm{d} u^{2}}{f(u)}+u^{2} \mathrm{~d} \Omega_{4}^{2}\right)\right]
$$

with $f(u)=1-u_{\kappa}^{3} / u^{3}$. We have two Euclidean compactified dimensions $t$ and $x_{4}: t \sim t+\beta$, $x_{4} \sim x_{4}+\beta_{4}$. For $T>T_{c}$, the role of $t$ and $x_{4}$ is interchanged and the deconfinement phase transition is described as a Hawking-Page geometrical transition where the cigar and cylinder geometries in $x_{4}$ and $t$ respectively are interchanged (Fig. 1).
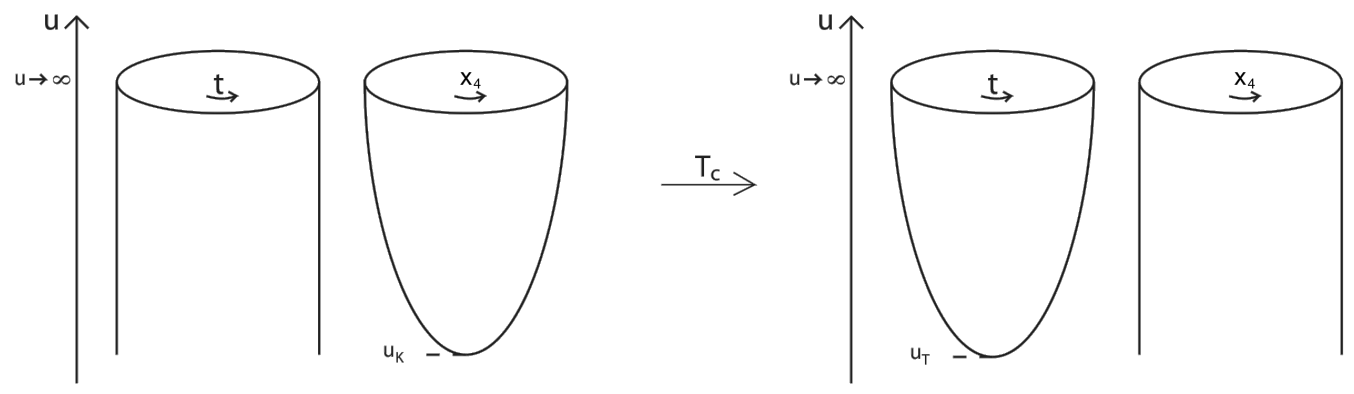

Figure 1: deconfinement phase transition and interchange of $x_{4}$ and $t$

We can now reinterpret this phase transition in terms of scalar fields. For $T<T_{c}$, the metric (7.1) describes a Euclidean black hole with Hawking "temperature" $1 / \beta_{4}$. The stretched membrane is described by a scalar field living in four dimensions $(\vec{x}, t)$. The formation of this "black hole" would correspond to the condensation of this scalar field. As the metric below $T_{c}$ is independent of $\beta$, it describes the quantum vacuum and the $4 \mathrm{D}$ scalar is a quantum field living in the vacuum. 
Above $T_{c}$, we have interchange of $x_{4}$ and $t$ and we have a black hole with Hawking temperature $1 / \beta$. The formation of this black hole is due to the condensation of a $3 \mathrm{D}$ thermal scalar $\left(x_{4}\right.$ being invisible to our world at low energies). So the Hawking-Page transition describing deconfinement can be interpreted as a change from a condensed 4D vacuum scalar field to a condensed 3D thermal scalar field. These scalar fields live close to the horizon and describe the strongly coupled infrared degrees of freedom of the plasma.

This scenario which is holography inspired, has been observed also in lattice simulations of pure Yang-Mills theory around $T_{c}$. Below $T_{c}$, magnetic strings percolate in $4 \mathrm{D}$ and induce confinement. They can be described by a $4 \mathrm{D}$ quantum condensed field living in the infrared. Above $T_{c}$, the magnetic strings change from virtual to real by becoming time oriented and wrapping around time. 4D confinement disappears and is replaced with spatial confinement by 3D percolation of magnetic strings. These types of percolation can be described on the lattice [15] with scalar order parameters which vanish in the UV. Again this points to the fact that that they can be holographically described by light scalar fields living close to the horizon.

\section{Conclusions}

Holographic models of the quark-gluon plasma point to the importance of light scalars living close to the horizon. These scalars become light by winding around compact directions and have a stringy origin. As has been suggested in many works [9], the formation of the horizon is due to condensation of these winding modes. We suggest in this paper that these light scalars can be also used to understand and describe the hydrodynamic behaviour of the stretched membrane and by the fluid/gravity correspondence, the hydrodynamic behaviour of the quark-gluon plasma. We pointed out that the basic scenario for deconfinement is a change from a condensed 4D quantum scalar to a condensed 3D thermal scalar field and that this precise scenario has been observed on the lattice. As suggested in [15], the importance of these light scalars can be checked on the lattice by measuring static correlators of momentum density.

\section{References}

[1] P. Kovtun, D.T. Son and A.O. Starinets, "Viscosity in strongly interacting quantum field theories from black hole physic”, Phys. Rev. Lett. 94 (2005) 111601, [arXiv:hep-th/0405231]; D.T. Son and A.O. Starinets, "Viscosity, black holes, and quantum field theory”, Ann. Rev. Nucl. Part. Sci. 57 (2007) 95, [arXiv:0704.0240 [hep-th]].

[2] A. Gorsky and V.I. Zakharov, "Magnetic strings in lattice QCD as nonabelian vortices", Phys. Rev. D77 (2008) 045017, [arXiv:0707.1284 [hep-th]]; M.N. Chernodub, A. Nakamura and V.I. Zakharov, "Manifestations of magnetic vortices in equation of state of Yang-Mills plasma", Phys. Rev. D78 (2008) 074021, [arXiv:0807.5012v2 [hep-lat]]; A.S. Gorsky, V.I. Zakharov and A.R. Zhitnitsky, "On classification of QCD defects via holography”, Phys. Rev. D79 (2009) 106003, [arXiv:0902.1842 [hep-ph]].

[3] M. Kruczenski and A. Lawrence, "Random walks and the Hagedorn transition", JHEP 0607031 (2006), [arXiv:hep-th/0508148] and references therein.

[4] J.L.F. Barbon and E. Rabinovici, “Touring the Hagedorn ridge”, [arXiv:hep-th/0407236v2] and references therein. 
[5] J. Polchinski, "Evaluation of the one loop string path integral", Commun. Math. Phys. 104 (1986) 37.

[6] B. McClain and B.D.B. Roth, "Modular invariance for interacting bosonic strings at finite temperature”, Commun. Math. Phys. 111 (1987) 539.

[7] B. Sathiapalan, "Vortices on the string world sheet and constraints on toral compactification", Phys. Rev. D35 (1987) 3277; Ya. I. Kogan, "Vortices on the world sheet and string's critical dynamics", JETP Lett. 45 (1987) 709.

[8] J.J. Atick and E. Witten, "The Hagedorn transition and the number of degrees of freedom of string theory”, Nucl. Phys. B310 (1988) 291.

[9] J.L.F. Barbon and E. Rabinovici, "Closed string tachyons and the Hagedorn transition in AdS space", JHEP 0203 (2002) 057, [arXiv:hep-th/0112173]; A. Adams, X. Liu, J. McGreevy, A. Saltman and E. Silverstein, "Things fall apart: topology change from winding tachyons", JHEP 0510 (2005) 033, [arXiv:hep-th/0502021]; G.T. Horowitz, "Tachyon condensation and black holes", JHEP 0508 (2005) 091; G.T. Horowitz and E. Silverstein, "The inside story: quasilocal tachyons and black holes", Phys. Rev. D73 (2006) 064016, [arXiv:hep-th/0601032].

[10] J. Polchinski, "String theory”, Cambridge Monographs on Mathematical Physics, Cambridge University Press (1998).

[11] H. Verschelde and V.I. Zakharov, in preparation.

[12] K.S. Thorne, R.H. Price and D.A. Macdonald, "Black holes and the membrane paradigm", Yale University Press (1986).

[13] L. Susskind and J. Uglum, "Black hole entropy in canonical quantum gravity and superstring theory”, Phys. Rev. D50 (1994) 2700, [arXiv:hep-th/9401070v3].

[14] E. Witten, "Anti-de Sitter space, thermal phase transition, and confinement in gauge theories", Adv. Theor. Math. Phys. 2 (1998) 505, [arXiv:hep-th/9803131]; E. Witten, "Theta dependence in the large N limit of four-dimensional gauge theories”, Phys. Rev. Lett. 81 (1998) 2862,

[arXiv:hep-th/9807109]; N. Itzhaki, J.M. Maldacena, J. Sonnenschein and Sh. Yankielowicz, "Supergravity and the large N limit of theories with sixteen supercharges", Phys. Rev. D58 (1998) 046004, [arXiv:hep-th/9802042]; T. Sakai and Sh. Sugimoto, "Low energy hadron physics in holographic QCD", Prog. Theor. Phys. 113 (2005) 843, [arXiv:hep-th/0412141]; O. Aharony, J. Sonnenschein and Sh. Yankielowicz, "A holographic model of deconfinement and chiral symmetry restoration", Annals Phys. 322 (2007) 1420, [arXiv:hep-th/0604161].

[15] H. Verschelde and V.I. Zakharov, "Effective scalar fields in Yang-Mills theories", see these proceedings "The many faces of $Q C D$ ". 